\title{
Four closely related equilibrated flux reconstructions for nonconforming finite elements *
}

\author{
Alexandre Ern ${ }^{\mathrm{a}}$, Martin Vohralík ${ }^{\mathrm{b}}$ \\ a Université Paris-Est, CERMICS, Ecole des Ponts ParisTech, 77455 Marne la Vallée, France \\ b INRIA Paris-Rocquencourt, B.P. 105, 78153 Le Chesnay, France \\ Received $* * * * *$; accepted after revision +++++ \\ Presented by $£ £ £ £ £$
}

\begin{abstract}
We consider the Crouzeix-Raviart nonconforming finite element method for the Laplace equation. We present four equilibrated flux reconstructions, by direct prescription or by mixed approximation of local Neumann problems, either relying on the original simplicial mesh only or employing a dual mesh. We show that all these reconstructions coincide provided the underlying system of linear algebraic equations is solved exactly. We finally consider an inexact algebraic solve, adjust the flux reconstructions, and point out the differences. To cite this article: A. Ern, M. Vohralik, C. R. Acad. Sci. Paris, Ser. I 340 (2012).
\end{abstract}

\section{Résumé}

Quatre reconstructions très proches de flux équilibrés pour les éléments finis non conformes. Nous étudions la méthode des éléments finis non conformes de Crouzeix et Raviart pour l'équation de Laplace. Nous introduisons quatre reconstructions équilibrées du flux, par prescription directe ou par une approximation mixte de problèmes locaux de Neumann, soit sur le maillage simplectique de départ, soit sur un maillage dual. Nous montrons que toutes ces reconstructions coïncident si le système d'équations linéaires associé est résolu exactement. Nous considérons enfin une solution algébrique inexacte, ajustons les reconstructions du flux et indiquons les différences entre les reconstructions. Pour citer cet article : A. Ern, M. Vohralík, C. R. Acad. Sci. Paris, Ser. I 340 (2012).

\section{Introduction}

We consider the Poisson problem for the Laplace equation: find $u: \Omega \rightarrow \mathbb{R}$ such that

$$
\begin{aligned}
-\Delta u=f & \text { in } \Omega, \\
u=0 & \text { on } \partial \Omega,
\end{aligned}
$$

where $\Omega \subset \mathbb{R}^{d}, d \geq 2$, is a polygonal domain (open, bounded, and connected set) and $f$ is for simplicity supposed piecewise constant on a matching simplicial mesh $\mathcal{T}_{h}$ of $\Omega$. We discretize (1) by means of the Crouzeix-Raviart nonconforming finite element method. Let $\mathcal{E}_{h}$ denote the faces of $\mathcal{T}_{h} ; \mathcal{E}_{h}^{\text {int }} \subset \mathcal{E}_{h}$ stands for interfaces and $\mathcal{E}_{h}^{\text {ext }} \subset \mathcal{E}_{h}$ for boundary faces. We associate with each $e \in \mathcal{E}_{h}^{\text {int }}$ the basis function $\psi_{e}$ which is piecewise affine on $\mathcal{T}_{h}$ and satisfies $\psi_{e}\left(\mathbf{x}_{e^{\prime}}\right)=\delta_{e, e^{\prime}}, e^{\prime} \in \mathcal{E}_{h}$, where $\mathbf{x}_{e}$ is the barycenter of the face $e$ and $\delta_{e, e^{\prime}}$ the Kronecker symbol. The Crouzeix-Raviart nonconforming finite element space is $V_{h}:=\operatorname{span}\left\{\psi_{e} ; e \in \mathcal{E}_{h}^{\text {int }}\right\}$ and the corresponding finite element method reads: find $u_{h} \in V_{h}$ such that

$$
\left(\nabla u_{h}, \nabla v_{h}\right)=\left(f, v_{h}\right) \quad \forall v_{h} \in V_{h} .
$$

^. This work was partly supported by the Groupement MoMaS (PACEN/CNRS, ANDRA, BRGM, CEA, EdF, IRSN) and by the ERT project "Enhanced oil recovery and geological sequestration of $\mathrm{CO}_{2}$ : mesh adaptivity, a posteriori error control, and other advanced techniques" (LJLL UPMC/IFPEN).

Email addresses: ern@cermics.enpc.fr (Alexandre Ern), martin.vohralik@inria.fr (Martin Vohralík). 
Here, $\nabla$ stands for the broken (elementwise) gradient operator and $(\cdot, \cdot)$ for the $L^{2}(\Omega)$ scalar product. Notice that $V_{h} \not \subset H_{0}^{1}(\Omega)$.

Following the early result of Prager and Synge and the concept of equilibrated fluxes, guaranteed and efficient a posteriori error estimates for conforming finite elements were obtained by Luce and Wohlmuth [10] and Braess and Schöberl [3], see also [5, 12, 7] and the references therein. In the context of nonconforming finite elements, similar results were obtained by Destuynder and Métivet [4], Ainsworth [1], Kim [9], and Braess [2]. An equilibrated flux reconstruction is a vector function $\boldsymbol{\sigma}_{h}$ belonging to $\mathbf{H}(\mathrm{div}, \Omega)$, typically built in some finite-dimensional mixed finite element space, constructed locally, designed to approximate $\sigma:=-\nabla u$, and satisfying

$$
\nabla \cdot \boldsymbol{\sigma}_{h}=f
$$

In the context of the nonconforming finite element method (2), equilibrated flux reconstructions lead to a guaranteed a posteriori error estimate of the form, see $[9,5]$,

$$
\left\|\nabla\left(u-u_{h}\right)\right\|^{2} \leq\left\|\nabla u_{h}+\sigma_{h}\right\|^{2}+\left\|\nabla\left(u_{h}-s_{h}\right)\right\|^{2}
$$

where $s_{h} \in H_{0}^{1}(\Omega)$ is an arbitrary potential reconstruction.

In this Note, we present four equilibrated flux reconstructions for nonconforming finite elements. The first one is the construction used in the a posteriori context in [4], while the three other ones are extensions of the constructions of $[10,3,12]$ to the nonconforming setting. Then, a little surprisingly, we show that these four constructions are all equivalent in the absence of algebraic errors, i.e., when the system of linear algebraic equations resulting from (2) is solved exactly. Finally, in the presence of algebraic errors, we show how to adjust the flux reconstructions and we indicate the differences among them.

\section{Four equilibrated flux reconstructions}

\subsection{Direct prescription on the original mesh}

Define $\left.\mathbf{f}_{h}(\mathbf{x})\right|_{K}:=\frac{\left.f\right|_{K}}{d}\left(\mathbf{x}-\mathbf{x}_{K}\right)$, with $\mathbf{x}_{K}$ the barycenter of $K \in \mathcal{T}_{h}$. Following [4], set

$$
\boldsymbol{\sigma}_{h}:=-\nabla u_{h}+\mathbf{f}_{h}
$$

It follows from the link of nonconforming to mixed finite elements by Marini [11] that $\boldsymbol{\sigma}_{h} \in \mathbf{R T N}_{0}\left(\mathcal{T}_{h}\right)$, where $\mathbf{R T N}_{0}\left(\mathcal{T}_{h}\right)$ is the lowest-order Raviart-Thomas-Nédélec mixed finite element space of functions $\mathbf{v}_{h} \in \mathbf{H}(\operatorname{div}, \Omega)$ such that $\left.\mathbf{v}_{h}\right|_{K} \in\left[\mathbb{P}_{0}(K)\right]^{d}+\mathbf{x} \mathbb{P}_{0}(K)$ for all $K \in \mathcal{T}_{h}$. By construction, (3) holds.

\subsection{Direct prescription on a dual mesh}

Following an idea in [10], see also [12, 7] and the references therein, we now present a construction relying on a dual mesh. For all $e \in \mathcal{E}_{h}$ and $K \in \mathcal{T}_{h}$ with $e \subset \partial K$, let $K_{e}$ be the sub-simplex of $K$ given by the face $e$ and the barycenter $\mathbf{x}_{K}$ of $K$. Let $\mathcal{S}_{h}$ be the mesh formed by the sub-simplices $K_{e}$ and let $\mathcal{D}_{h}$ be the dual mesh regrouping for each $e \in \mathcal{E}_{h}$ the two (or one for boundary faces) simplices $K_{e}$ which share $e$ (denoted by $D_{e}$ ). It is easily verified that (2) is equivalent to looking for $u_{h} \in V_{h}$ such that

$$
-\left\langle\nabla u_{h} \cdot \mathbf{n}_{D_{e}}, 1\right\rangle_{\partial D_{e}}=(f, 1)_{D_{e}} \quad \forall e \in \mathcal{E}_{h}^{\text {int }},
$$

which corresponds to the face-centered finite volume method. Define $\boldsymbol{\sigma}_{h} \in \mathbf{R T N}_{0}\left(\mathcal{S}_{h}\right)$ by

$$
\begin{aligned}
\left.\boldsymbol{\sigma}_{h} \cdot \mathbf{n}_{D_{e}}\right|_{\partial D_{e} \backslash \partial \Omega} & :=-\left.\nabla u_{h} \cdot \mathbf{n}_{D_{e}}\right|_{\partial D_{e} \backslash \partial \Omega} & & \forall e \in \mathcal{E}_{h}, \\
\left.\boldsymbol{\sigma}_{h} \cdot \mathbf{n}_{K_{e}}\right|_{e} & :=|e|^{-1}\left\{(f, 1)_{K_{e}}-\left\langle\boldsymbol{\sigma}_{h} \cdot \mathbf{n}_{K_{e}}, 1\right\rangle_{\partial K_{e} \backslash e}\right\} & & \forall e \in \mathcal{E}_{h}, K_{e} \subset D_{e},
\end{aligned}
$$

where $\mathbf{n}_{D_{e}}$ and $\mathbf{n}_{K_{e}}$ denote outward normals of $D_{e}$ and $K_{e}$, respectively. Here, (7a) prescribes the normal component of $\boldsymbol{\sigma}_{h}$ on all faces of the mesh $\mathcal{S}_{h}$ which lie on the boundary of some $D_{e} \in \mathcal{D}_{h}$ but not on $\partial \Omega$ (and thus inside the elements of $\mathcal{T}_{h}$ ), whereas (7b) prescribes the normal component on those faces of the mesh $\mathcal{S}_{h}$ which are faces of $\mathcal{T}_{h}$. It follows from (6) and (7a) that the definition (7b) is independent of the choice of $K_{e} \subset D_{e}$; (7b) fixes the normal component of $\boldsymbol{\sigma}_{h}$ on the faces of $\mathcal{T}_{h}$ so that (3) holds.

\subsection{Mixed approximation of local Neumann problems with scheme-given normal flux on dual mesh}

The next construction is tightly linked to the construction of $\S 2.2$, while adopting a different viewpoint following $[10,5,12,7]$. For a given dual volume $D_{e} \in \mathcal{D}_{h}$, let $\mathcal{S}_{D_{e}}$ stand for the submesh of the dual volume $D_{e}$ by the simplices of $\mathcal{S}_{h}$. For all $e \in \mathcal{E}_{h}$, define the space

$$
\mathbf{R T N}_{0}^{\mathrm{N}}\left(\mathcal{S}_{D_{e}}\right):=\left\{\mathbf{v}_{h} \in \mathbf{R} \mathbf{T N}_{0}\left(\mathcal{S}_{D_{e}}\right) ;\left.\mathbf{v}_{h} \cdot \mathbf{n}_{D_{e}}\right|_{\partial D_{e} \backslash \partial \Omega}=-\left.\nabla u_{h} \cdot \mathbf{n}_{D_{e}}\right|_{\partial D_{e} \backslash \partial \Omega}\right\},
$$


spanned by Raviart-Thomas-Nédélec vector functions with normal component over $\partial D_{e} \backslash \partial \Omega$ given by (7a). We construct $\boldsymbol{\sigma}_{h} \in \mathbf{R T N}_{0}\left(\mathcal{S}_{h}\right)$, while fixing the remaining degrees of freedom by

$$
\left.\boldsymbol{\sigma}_{h}\right|_{D_{e}}:=\arg \inf _{\mathbf{v}_{h} \in \mathbf{R T N}_{0}^{N}\left(\mathcal{S}_{D_{e}}\right), \nabla \cdot \mathbf{v}_{h}=f}\left\|\nabla u_{h}+\mathbf{v}_{h}\right\|_{D_{e}} \quad \forall e \in \mathcal{E}_{h},
$$

instead of $(7 \mathrm{~b})$. Note that this complementary energy minimization problem locally minimizes the size of the first estimator in (4). Let $\mathbf{R T N}_{0}^{\mathrm{N}, 0}\left(\mathcal{S}_{D_{e}}\right)$ be defined as (8), but with the normal flux condition $\left.\mathbf{v}_{h} \cdot \mathbf{n}_{D_{e}}\right|_{\partial D_{e} \backslash \partial \Omega}=0$. Finally, let $\mathbb{P}_{0}^{*}\left(\mathcal{S}_{D_{e}}\right)$ be spanned by piecewise constants on $\mathcal{S}_{D_{e}}$ with zero mean value on the dual cell $D_{e}$ when $e \in \mathcal{E}_{h}^{\text {int }}$ and by constants when $e \in \mathcal{E}_{h}^{\text {ext }}$. Problem (9) is equivalent, cf. [5], to finding $\left.\boldsymbol{\sigma}_{h}\right|_{D_{e}} \in \mathbf{R T N}_{0}^{\mathrm{N}}\left(\mathcal{S}_{D_{e}}\right)$ and $\left.q_{h}\right|_{D_{e}} \in \mathbb{P}_{0}^{*}\left(\mathcal{S}_{D_{e}}\right)$ such that

$$
\begin{array}{rlrl}
\left(\boldsymbol{\sigma}_{h}, \mathbf{v}_{h}\right)_{D_{e}}-\left(q_{h}, \nabla \cdot \mathbf{v}_{h}\right)_{D_{e}} & =-\left(\nabla u_{h}, \mathbf{v}_{h}\right)_{D_{e}} & & \forall \mathbf{v}_{h} \in \mathbf{R T N}_{0}^{\mathrm{N}, 0}\left(\mathcal{S}_{D_{e}}\right), \\
\left(\nabla \cdot \boldsymbol{\sigma}_{h}, \phi_{h}\right)_{D_{e}}=\left(f, \phi_{h}\right)_{D_{e}} & \forall \phi_{h} \in \mathbb{P}_{0}^{*}\left(\mathcal{S}_{D_{e}}\right) .
\end{array}
$$

(10) is the lowest-order Raviart-Thomas-Nédélec mixed finite element approximation of a local inhomogeneous Neumann problem on the dual volumes $D_{e}, e \in \mathcal{E}_{h}^{\text {int }}$. For $e \in \mathcal{E}_{h}^{\text {ext }}$, this is a local problem with inhomogeneous Neumann boundary condition on that part of $\partial D_{e}$ which lies inside $\Omega$ and homogeneous Dirichlet boundary condition on $\partial D_{e} \cap \partial \Omega$. For $e \in \mathcal{E}_{h}^{\text {int }}$, the compatibility of the Neumann condition with the source term $f$ is nothing but (6). Thus, the well-posedness of (10) is standard.

\subsection{Mixed approximation of homogeneous local Neumann problems and partition of unity}

Finally, we rewrite differently and transfer to the nonconforming setting the construction of [3]. For all $e \in \mathcal{E}_{h}$, let $\mathcal{T}_{e}$ collect the two (or one for boundary faces) mesh elements in $\mathcal{T}_{h}$ of which $e$ is a face. For $e \in \mathcal{E}_{h}$, denote $\mathbf{R T N}_{0}^{\mathrm{N}, 0}\left(\mathcal{T}_{e}\right)$ the subspace of $\mathbf{R T N}_{0}\left(\mathcal{T}_{e}\right)$ with zero normal flux through $\partial \mathcal{T}_{e}$ for $e \in \mathcal{E}_{h}^{\text {int }}$ and through that part of $\partial \mathcal{T}_{e}$ which lies inside $\Omega$ for $e \in \mathcal{E}_{h}^{\text {ext }}$. Let $\mathbb{P}_{0}^{*}\left(\mathcal{T}_{e}\right)$ be spanned by piecewise constants on $\mathcal{T}_{e}$ with zero mean on $\mathcal{T}_{e}$ when $e \in \mathcal{E}_{h}^{\text {int }}$; when $e \in \mathcal{E}_{h}^{\text {ext }}$, the mean value condition is not imposed. Recall that $\psi_{e}$ stands for the Crouzeix-Raviart basis function (we will use it now also for $e \in \mathcal{E}_{h}^{\text {ext }}$ ). Define $\boldsymbol{\sigma}_{h}^{e} \in \mathbf{R T N}_{0}^{\mathrm{N}, 0}\left(\mathcal{T}_{e}\right)$ and $q_{h}^{e} \in \mathbb{P}_{0}^{*}\left(\mathcal{T}_{e}\right)$ by

$$
\begin{array}{ll}
\left(\boldsymbol{\sigma}_{h}^{e}, \mathbf{v}_{h}\right)_{\mathcal{T}_{e}}-\left(q_{h}^{e}, \nabla \cdot \mathbf{v}_{h}\right)_{\mathcal{T}_{e}}=-\left(\psi_{e} \nabla u_{h}, \mathbf{v}_{h}\right)_{\mathcal{T}_{e}} & \forall \mathbf{v}_{h} \in \mathbf{R T N}_{0}^{\mathrm{N}, 0}\left(\mathcal{T}_{e}\right), \\
\left(\nabla \cdot \boldsymbol{\sigma}_{h}^{e}, \phi_{h}\right)_{\mathcal{T}_{e}}=\left(f \psi_{e}, \phi_{h}\right)_{\mathcal{T}_{e}}-\left(\nabla u_{h} \cdot \nabla \psi_{e}, \phi_{h}\right)_{\mathcal{T}_{e}} & \forall \phi_{h} \in \mathbb{P}_{0}^{*}\left(\mathcal{T}_{e}\right) .
\end{array}
$$

Then, set $\boldsymbol{\sigma}_{h}:=\sum_{e \in \mathcal{E}_{h}} \boldsymbol{\sigma}_{h}^{e}$. Note that the problems (11) are well-posed. Indeed, they lead to square linear systems such that setting their right-hand side to zero yields a zero solution. For interfaces $e \in \mathcal{E}_{h}^{\text {int }}$, they represent a local homogeneous Neumann problem on $\mathcal{T}_{e}$, whereas for boundary faces $e \in \mathcal{E}_{h}^{\text {ext }}$, this is a local homogeneous Neumann/Dirichlet (on $\partial \Omega$ ) problem on $\mathcal{T}_{e}$. Moreover, on $e \in \mathcal{E}_{h}^{\text {int }}$, the Neumann compatibility condition on the data is satisfied ( $\operatorname{set} \phi_{h}=1$ on $\mathcal{T}_{e}$ in (11b) and use (2) with $v_{h}=\psi_{e}$ ).

\section{Equivalence of the four flux reconstructions}

Theorem 3.1 (Equivalence of (5), (7), (9), and (11)) The constructions of Sections 2.1-2.4 yield the same equilibrated flux reconstruction $\boldsymbol{\sigma}_{h}$.

Proof: In view of the term $\left(\mathbf{x}-\mathbf{x}_{K}\right)$ in the definition of $\mathbf{f}_{h}, \mathbf{f}_{h} \cdot \mathbf{n}_{D_{e}}=0$ on $\partial D_{e}, e \in \mathcal{E}_{h}^{\text {int }}$. Hence, $\boldsymbol{\sigma}_{h}$ of (5) satisfies (7a). Moreover, (5) immediately implies (3), whence (7b) follows by the Green theorem. Thus, (5) and (7) are equivalent. Next, the normal boundary conditions on $\partial D_{e}, e \in \mathcal{E}_{h}^{\text {int }}$, in (7) and (10) are the same. Both $(7 \mathrm{~b})$ and $(10 \mathrm{~b})$ then fix the remaining degrees of freedom (the fluxes over $e \in \mathcal{E}_{h}$ ) such that (3) holds. Hence, (7) and (10) are equivalent. Let finally $e \in \mathcal{E}_{h}^{\text {int }}$. Owing to the Neumann compatibility condition, we can take $\phi_{h}=1$ on one simplex $K$ of $\mathcal{T}_{e}$ and $\phi_{h}=0$ on the other one as the test function in (11b). Using that $\left.f\right|_{K}$ is constant and the side quadrature formula for the first term on the right-hand side of (11b) and the Green formula and some elementary calculus for the two other terms, we arrive at $\left\langle\boldsymbol{\sigma}_{h}^{e} \cdot \mathbf{n}_{K}, 1\right\rangle_{e}=\left.f\right|_{K} \frac{|K|}{d+1}-\left\langle\nabla u_{h} \cdot \mathbf{n}_{K}, 1\right\rangle_{e}$, which fixes the flux of $\boldsymbol{\sigma}_{h}$ through $e$ in the same way as (5). For $e \in \mathcal{E}_{h}^{\text {ext }}$, we proceed similarly. Thus all the reconstructions are equivalent.

Remark 1 (Local efficiency) For a suitable choice of the potential reconstruction $s_{h}$, following [1, 9, 2], local efficiency holds in the sense that there exists a constant $C>0$ only depending on the space dimension $d$ and on the shape regularity of $\mathcal{T}_{h}$ such that

$$
\left(\left\|\nabla u_{h}+\sigma_{h}\right\|_{K}^{2}+\left\|\nabla\left(u_{h}-s_{h}\right)\right\|_{K}^{2}\right)^{\frac{1}{2}} \leq C\left\|\nabla\left(u-u_{h}\right)\right\|_{\mathfrak{T}_{K}}
$$

for all $K \in \mathcal{T}_{h}$, where $\mathfrak{T}_{K}$ stands for all the elements sharing a vertex with $K$. 


\section{Taking into account the algebraic error}

In practice, solving the linear system associated with (2) exactly (to computer working precision) is quite demanding. Moreover, such an effort is unnecessary in view of the unavoidable presence of the discretization error $\left\|\nabla\left(u-u_{h}\right)\right\|$. Guaranteed a posteriori error estimates not requiring (2) or (3) and distinguishing the discretization and algebraic errors are now available, see $[8,6]$ and the references therein, and we present them here in the Crouzeix-Raviart context.

Consider $\psi_{e}, e \in \mathcal{E}_{h}^{\text {int }}$, as test function in (2). Applying an iterative solver to the resulting system of linear algebraic equations, we obtain on step $i \geq 0$ of this solver a discrete potential $u_{h}^{i} \in V_{h}$ such that

$$
\left(\nabla u_{h}^{i}, \nabla \psi_{e}\right)=\left(f, \psi_{e}\right)-R_{e}^{i} \quad \forall e \in \mathcal{E}_{h}^{\text {int }},
$$

where $R^{i}=\left\{R_{e}^{i}\right\}_{e \in \mathcal{E}_{h}^{\text {int }}}$ is the algebraic residual vector. For convenience, we set $R_{e}^{i}:=0$ for all $e \in \mathcal{E}_{h}^{\text {ext }}$.

In order to extend the results of Section 2 to this context, a key idea is to relax (3) into a quasiequilibrated flux reconstruction such that, at step $i \geq 0$,

$$
\nabla \cdot \boldsymbol{\sigma}_{h}^{i}=f-\rho_{h}^{i}
$$

with the algebraic remainder function $\rho_{h}^{i}$ linked to the algebraic residual vector $R^{i}$. In extension of the approach of $\S 2.1$, we set

$$
\left.\boldsymbol{\sigma}_{h}^{i}\right|_{K}:=\left.\left(-\nabla u_{h}^{i}+\mathbf{f}_{h}\right)\right|_{K}-\sum_{e \in \mathcal{E}_{K}}\left|\mathcal{T}_{e}\right|^{-1} \frac{R_{e}^{i}}{d}\left(\mathbf{x}-\mathbf{a}_{K, e}\right) \quad \forall K \in \mathcal{T}_{h},
$$

where $\mathcal{E}_{K}$ regroups the faces of $K$ and $\mathbf{a}_{K, e}$ is the vertex of $K$ opposite to $e$. In particular, there still holds $\boldsymbol{\sigma}_{h}^{i} \in \mathbf{R T N}_{0}\left(\mathcal{T}_{h}\right)$ and $\rho_{h}^{i}$ in (13) is piecewise constant on $\mathcal{T}_{h}$ with $\left.\rho_{h}^{i}\right|_{K}=\sum_{e \in \mathcal{E}_{K}}\left|\mathcal{T}_{e}\right|^{-1} R_{e}^{i}$. Equivalently, (14) also results from extending the approach of $\S 2.4$ by subtracting $\left|\mathcal{T}_{e}\right|^{-1}\left(R_{e}^{i}, \phi_{h}\right)_{\mathcal{T}_{e}}$ to the right-hand side of (11b). Replacing in (14) $\left(\mathbf{x}-\mathbf{a}_{K, e}\right)$ by $\left.\left(\mathbf{x}-\mathbf{x}_{K}\right)\right|_{K \cap D_{e}}$ and $\left|\mathcal{T}_{e}\right|^{-1}$ by $\left|D_{e}\right|^{-1}$ leads instead to a construction extending those of $\S 2.2$ and $\S 2.3$. Specifically, $|e|^{-1}\left|D_{e}\right|^{-1}\left(R_{e}^{i}, 1\right)_{K_{e}}$ is subtracted to the right-hand side of (7b), and the constraint in (9) is replaced by $\nabla \cdot \mathbf{v}_{h}=f-\left|D_{e}\right|^{-1} R_{e}^{i}$. Such a $\boldsymbol{\sigma}_{h}^{i}$ now belongs to $\mathbf{R T N}_{0}\left(\mathcal{S}_{h}\right)$, but not to $\mathbf{R T N}_{0}\left(\mathcal{T}_{h}\right)$, and localizes more precisely the algebraic error around the interfaces since $\rho_{h}^{i}$ in (13) is now piecewise constant on $\mathcal{D}_{h}$ with $\left.\rho_{h}^{i}\right|_{D_{e}}=\left|D_{e}\right|^{-1} R_{e}^{i}$. More details can be found in $[6]$.

\section{References}

[1] Mark Ainsworth. Robust a posteriori error estimation for nonconforming finite element approximation. SIAM J. Numer. Anal., 42(6):2320-2341, 2005.

[2] Dietrich Braess. An a posteriori error estimate and a comparison theorem for the nonconforming $P_{1}$ element. Calcolo, 46(2):149-155, 2009.

[3] Dietrich Braess and Joachim Schöberl. Equilibrated residual error estimator for edge elements. Math. Comp., 77(262):651-672, 2008.

[4] Philippe Destuynder and Brigitte Métivet. Explicit error bounds for a nonconforming finite element method. SIAM J. Numer. Anal., 35(5):2099-2115, 1998.

[5] Alexandre Ern and Martin Vohralík. Flux reconstruction and a posteriori error estimation for discontinuous Galerkin methods on general nonmatching grids. C. R. Math. Acad. Sci. Paris, 347(78):441-444, 2009.

[6] Alexandre Ern and Martin Vohralík. Adaptive inexact Newton methods with a posteriori stopping criteria for nonlinear diffusion PDEs. HAL Preprint 00681422 v2, submitted for publication, 2012.

[7] Antti Hannukainen, Rolf Stenberg, and Martin Vohralík. A unified framework for a posteriori error estimation for the Stokes problem. Numer. Math., 2012. DOI 10.1007/s00211-012-0472-x.

[8] Pavel Jiránek, Zdeněk Strakoš, and Martin Vohralík. A posteriori error estimates including algebraic error and stopping criteria for iterative solvers. SIAM J. Sci. Comput., 32(3):1567-1590, 2010.

[9] Kwang Y. Kim. A posteriori error analysis for locally conservative mixed methods. Math. Comp., 76(257):43-66, 2007.

[10] Robert Luce and Barbara I. Wohlmuth. A local a posteriori error estimator based on equilibrated fluxes. SIAM J. Numer. Anal., 42(4):1394-1414, 2004.

[11] L. Donatella Marini. An inexpensive method for the evaluation of the solution of the lowest order Raviart-Thomas mixed method. SIAM J. Numer. Anal., 22(3):493-496, 1985.

[12] Martin Vohralík. Guaranteed and fully robust a posteriori error estimates for conforming discretizations of diffusion problems with discontinuous coefficients. J. Sci. Comput., 46(3):397-438, 2011. 